\title{
Pigment epithelium-derived factor downregulates vascular endothelial growth factor (VEGF) expression and inhibits VEGF-VEGF receptor 2 binding in diabetic retinopathy
}

\author{
Sarah X Zhang, Joshua J Wang, Guoquan Gao', Kyoungmin Parke and Jian-xing Ma \\ Department of Medicine, Department of Cell Biology, University of Oklahoma Health Sciences Center, 941 Stanton L. Young Blvd., Oklahoma City, Oklahoma, USA \\ 'Department of Biochemistry, Zhongshan University, Guangzhou, China \\ (Requests for offprints should be addressed to J-x Ma; Email: jian-xing-ma@ ouhsc.edu)
}

\begin{abstract}
It has been shown that the balance between vascular endothelial growth factor (VEGF), a major angiogenic stimulator, and pigment epithelium-derived factor (PEDF), a potent angiogenic inhibitor, is critical for the regulation of vascular permeability and angiogenesis. However, the regulation of the balance is largely unclear. The present study demonstrated that there is a reciprocal interaction between VEGF and PEDF in the retina. PEDF significantly decreased VEGF expression in both retinal capillary endothelial cells (RCEC) and Müller cells. This PEDF effect was confirmed in the retina of rats with oxygen-induced retinopathy. Silencing of the PEDF gene by siRNA in Müller cells resulted in a significant upregulation of VEGF expression at both the RNA and protein levels, suggesting that PEDF is an endogenous negative regulator of VEGF. The further study of the mechanism showed that PEDF inhibited hypoxia-induced increases in VEGF promoter activity, HIF-1 nuclear translocation and mitogen activated protein kinase phosphorylation. These results suggest that PEDF inhibits VEGF expression at the transcriptional level. In addition, PEDF effectively inhibited VEGF binding to RCEC. Moreover, in vitro receptor-binding assay demonstrated that PEDF competed with VEGF for binding to VEGF receptor 2, which may represent a new mechanism for PEDF activity. On the other hand, VEGF significantly downregulated PEDF expression in RCEC, but not in retinal Müller cells, suggesting a VEGF receptormediated process. These results suggest that the reciprocal regulation between VEGF and PEDF may play a role in angiogenic control. The decrease in PEDF levels in the retina is at least partially responsible for the increase in VEGF expression and subsequent vascular leakage and neovascularization in diabetes.
\end{abstract}

Journal of Molecular Endocrinology (2006) 37, 1-12

\section{Introduction}

The blood-retinal barrier (BRB) breakdown and retinal neovascularization $(\mathrm{NV})$ are the major retinal vascular dysfunctions leading to vision loss in a variety of retinal diseases such as diabetic retinopathy (DR) (Bresnick 1986, Frank 1991, Aiello et al. 1997, Pelzek \& Lim 2002). Although the exact pathogenic mechanisms are uncertain, it is widely accepted that the two counterbalancing systems, angiogenic stimulators such as vascular endothelial growth factor (VEGF) and angiogenic inhibitors such as angiostatin and pigment epithelium-derived factor (PEDF), play important roles in the regulation of vascular functions (Pierce et al. 1995, Dawson et al. 1999, Gao et al. 2001, Gao et al. 2002a). Under normal conditions, there is a balance between these two systems, which is essential for maintaining the quiescence of retinal vasculature and integrity of the BRB (Bussolino et al. 1997, Miller et al. 1997). Under some pathological conditions, such as DR, this balance is disturbed due to the overproduction of angiogenic stimulators and the decreased production of angiogenic inhibitors, which subsequently lead to the BRB breakdown and the overproliferation of capillary endothelial cells, resulting in retinal NV (Forsythe et al. 1996, Miller et al. 1997, Gao et al. 2001, Gao \& Ma 2002).

VEGF is a major angiogenic factor, playing a crucial role in normal and pathological angiogenesis (Pe'er et al. 1995, Aiello 1996, Aiello \& Wong 2000, Dvorak 2000). VEGF is also referred to as vascular permeability factor (VPF) based on its ability to induce vascular hyper-permeability (Dvorak et al. 1995). In the early stage of streptozotocin (STZ)-induced diabetes, significant increases in retinal VEGF levels have been found to correlate with retinal vascular permeability (Antonetti et al. 1998). This early BRB breakdown can be successfully prevented by VEGF TrapA $A_{40}$, a soluble VEGF receptor fms-like tyrosine kinase $(\mathrm{Flt}) / \mathrm{F}_{\mathrm{c}}$ chimera (Qaum et al. 2001). In the oxygen-induced retinopathy (OIR), a commonly used model for proliferative diabetic retinopathy (PDR), retinal VEGF levels are significantly elevated, which correlates with the retinal NV progression (Gao et al. 2002b). The VEGF 
levels decline to the normal level when the regression of NV occurs (Gao et al. 2002b). These findings suggest that VEGF is a key mediator of both vascular hyperpermeability and retinal NV.

PEDF is a neurotrophic factor and a potent angiogenic inhibitor, originally identified from cultured retinal pigment epithelial (RPE) cells (TombranTink et al. 1991, Dawson et al. 1999, Tombran-Tink \& Barnstable 2003a). Previously, we have demonstrated that, in OIR rats, PEDF levels in the retina are significantly decreased in contrast to the increased VEGF levels, which leads to an increased retinal VEGF/ PEDF ratio (Gao et al. 2001). This disturbed balance correlates with the formation and progression of retinal NV (Gao et al. 2001, 2002b). The decrease of PEDF is also observed in the vitreous and aqueous humor of the patients with active DR and diabetic macular edema (DME) (Ogata et al. 2001b, 2002, Boehm et al. 2003). After successful laser treatment, the PEDF levels in the vitreous were elevated, correlating with the regression of retinal NV (Ogata et al. 2001a).

Although the functions of PEDF and VEGF have been well studied in the eye, the interactions between these two counteracting angiogenic factors are largely unclear. Based on the solid evidence showing the inverse correlation between PEDF and VEGF levels in a variety of vascular diseases, we hypothesize that there exists a reciprocal regulation between these two major angiogenic regulators (Gao et al. 2001, Ohno-Matsui et al. 2001, Doll et al. 2003, Kim et al. 2003). In this study, we tested this hypothesis and explored the mechanisms by which PEDF regulates VEGF in retinal cells.

\section{Materials and methods}

\section{Experimental animals}

Brown Norway (BN) rats were purchased from Charles River Laboratories (Wilmington, MA, USA). Care, use and treatment of all animals in this study were in strict agreement with the guidelines in the Care and Use of Laboratory Animals set forth by the University of Oklahoma.

\section{Experimental reagents}

Recombinant human PEDF was purchased from BioProducts MD, LLC (Middletown, MD, USA). Recombinant human VEGF $_{165}$ was purchased from PeproTech Inc. (Rocky Hill, NJ, USA). $\mathrm{CoCl}_{2}$ and BSA were purchased from Sigma.

\section{Development of OIR rat model}

OIR rat model was established as described previously (Gao et al. 2001, Zhang et al. 2004, 2005b). Briefly, newborn $\mathrm{BN}$ rats were exposed to $75 \%$ oxygen at postnatal day 7 (P7) for 5 days and returned to room air to develop retinopathy. The intravitreal injection was given at P14 and the eyeball was enucleated at P16.

\section{Cell culture}

Primary bovine retinal capillary endothelial cells (RCEC) were isolated and cultured in DMEM supplied with $10 \%$ human serum as described previously (Gao et al. 2003). A rat Müller cell line (rMC-1) was also used in the experiments. After reaching $80 \%$ confluence, cells were exposed to serum-free medium for $12 \mathrm{~h}$ before the treatments with desired reagents.

\section{Western blot analysis of VEGF and PEDF}

Western blot analysis was performed as described previously (Gao et al. 2002a). Briefly, $50 \mu \mathrm{g}$ total protein from each sample were blotted with primary antibodies: anti-VEGF (1:500, Santa Cruz Biotechnology, Inc., Santa Cruz, CA, USA) and anti-PEDF (1:1000, Chemicon Inc., Temecula, CA, USA). The same membrane was stripped and reblotted with an anti- $\beta$-actin antibody for normalization.

\section{Quantification of VEGF and PEDF by ELISA}

The protein concentration was measured with the BioRad DC protein assay. The concentrations of VEGF and PEDF in the cell culture medium were measured using ELISA kits specific for VEGF (R\&D Systems, Minneapolis, MN, USA) and PEDF (Chemicon Inc.) according to the manufacturers' instructions.

\section{siRNA transfection in rat retinal Müller cells}

PEDF siRNA was synthesized using the silencer siRNA construction kit (Ambion, Austin, TX, USA). The following DNA templates were used to synthesize double-stranded PEDF, siRNA: sense, GGA UUU CUA CUU GGA UGA ATT, and anti-sense, UUC AUC CAA GUA GAA AUC. Transfection was performed with the siPORT lipid transfection reagent (Ambion) following the manufacturer's instructions.

\section{Semi-quantitative RT-PCR analysis}

Total RNA was extracted using the RNeasy miniisolation kit (Qiagen). PCR was performed as described previously (Shao et al. 2004), using PEDF-specific primers: forward primer, 5'-GTC ACT GCC CGC CAG AAG AAC-3 ${ }^{\prime}$, and reverse primer, $5^{\prime}$-TGT TAC CCA CTG CCC CTT GAA-3'. Specific PCR products were examined by electrophoresis in $1 \%$ agarose gel. 


\section{Real time RT-PCR analysis}

Primers specific for VEGF (VEGF-F, $5^{\prime}$-ggccaactacaacttcttcc-3' ${ }^{\prime}$, VEGF-R, $5^{\prime}$-ccatcatggattcgagaaaa-3') were used for real-time RT-PCR. The PCR was performed using GeneAmp RNA PCR kit and SYBR Green PCR Master Mix (Applied Biosystems, Foster City, CA, USA). The average $C_{\mathrm{T}}$ (threshold cycle) of fluorescence units was used to analyze the mRNA levels. The VEGF mRNA levels were normalized by $18 \mathrm{~S}$ ribosomal RNA levels. Quantification was calculated as: mRNA levels (percent of control $)=2^{\Delta\left(C_{\mathrm{T}}\right)}$ with $\Delta C_{\mathrm{T}}=C_{\mathrm{T}, \mathrm{VEGF}}-C_{\mathrm{T}, 18 \mathrm{~S} \mathrm{RNA}}$ and $\left(\Delta C_{\mathrm{T}}\right)=\Delta C_{\mathrm{T}}$ control $-\Delta C_{\mathrm{T}}$, treatment.

\section{Transient transfection and dual luciferase assay}

D407 cells, a cell-line derived from human RPE cells, were seeded onto 12-well culture plates at a density of $1.0 \times 10^{5}$ cells/well and grown for $24 \mathrm{~h}$. The VEGF promoter-luciferase reporter in the pGal3 vector was transfected into the cells using Fugene 6 transfection reagent (Roche) according to the manufacturer's instructions. In the case of co-transfection experiments, pRL-TK and pGal3-VEGF promoter constructs were cotransfected at a ratio of 3:2. Constructs of pGal3 (+) and pGal3 (-) were used as positive and negative control respectively. Eighteen hours following the transfection, the cells were exposed to $200 \mu \mathrm{M} \mathrm{CoCl} 2$ in the presence or absence of $160 \mathrm{nM}$ PEDF for $6 \mathrm{~h}$. Then the cells were washed with PBS, and lysed by passive lysis buffer (Promega). The cell lysate was collected and incubated with the luciferase assay reagent (Promega). Luminescence was measured by a microplate luminometer (Berthold Detection Systems, Pforzheim, Germany). The promoter activity was normalized by the Renilla reniformis luciferase from pRL-TK.

\section{Analysis of hypoxia-induced factor- $\alpha$ (HIF-1 $\alpha$ ) nuclear translocation}

Cultured RCEC were trypsinized, counted and washed three times in PBS. Nuclear and cytoplasmic proteins were extracted using NE-PER Nuclear and Cytoplasmic extraction kit (Pierce, Rockford, IL, USA) according to the manufacturer's protocol. The same amount of nuclear and cytoplasmic proteins was applied to Western blot analysis using an anti-HIF-1 antibody (Santa Cruz Biotechnology, Inc.) at a 1:500 dilution.

\section{Determination of MAP kinase activation}

The total proteins from the cultured RCEC or dissected retinas from OIR rats were isolated on 12\% SDS-PAGE. The phosphorylation of $\mathrm{p} 42 / \mathrm{p} 44$ was measured using a phosphorylation-specific antibody (1:1000, Santa Cruz
Biotechnology, Inc.) and normalized by the total p42/p44 levels.

\section{VEGF-binding assay in retinal endothelial cells}

VEGF (PeproTech) was labeled with ${ }^{125} \mathrm{I}$ using the Chloromine $\mathrm{T}^{125}$ I labeling kit (ICN Pharmaceuticals, Inc., Costa Mesa, CA, USA) following the manufacturer's protocol as described previously (Gotte et al. 2002, Gao et al. 2003). The same amount of ${ }^{125}$ I-VEGF (4 nM) was incubated with primary RCEC for $1 \mathrm{~h}$. Unbound ${ }^{125}$ I-VEGF was removed by four washes. The cells were then lysed, and the ${ }^{125}$ I-VEGF bound to the cells was quantified by a $\gamma$-counter, calculated to molar concentration based on VEGF standards.

\section{In vitro VEGF receptor-binding assay}

Microtiter plates (Corning Inc., Corning, NY, USA) with 96 wells were coated with $5 \mathrm{nM}$ carrier-free human recombinant VEGF receptor (KDR)/IgG Fc (R\&D Systems) in PBS overnight at $4{ }^{\circ} \mathrm{C}$. The wells were then washed three times with PBS containing $0.1 \%$ (v/v) Tween 20 (PBS-T) and blocked with 1\% BSA in PBS for $2 \mathrm{~h}$. The plates were then incubated for $2 \mathrm{~h}$ with $1 \mathrm{nM}$ recombinant human $\mathrm{VEGF}_{165}$ in the presence of various concentrations of PEDF or BSA, ranging from 0.1 to $1000 \mathrm{nM}$ as control. After three washes, a biotinylated anti-VEGF antibody (R\&D Systems) was added to the wells and incubated for another $2 \mathrm{~h}$, followed by incubation with horseradish peroxidase-conjugated streptavidin for $20 \mathrm{~min}$. Then the color reagents were added, and the results were measured by a microplate reader (Perkin-Elmer Wallac, Inc., Boston, MA, USA). All the experiments were carried out in triplicate and repeated three times.

\section{Statistical analysis}

Statistical analysis employed the Student's $t$-test. Statistical difference was considered significant at $P<0 \cdot 05$.

\section{Results}

PEDF decreased VEGF levels in cultured RCEC and Müller cells under normal and hypoxic conditions. RCEC is the major component of the BRB and serves as the primary target of VEGF (Hatcher et al. 1998). Cultured primary RCEC have been shown to produce high levels of VEGF (Simorre-Pinatel et al. 1994). The retinal Müller cell has been recognized as a major source of VEGF in the retina (Hata et al. 1995). We first determined the effect of PEDF on the expression of VEGF in cultured RCEC and Müller cells. Under normal culture conditions, VEGF is 
detected in both RCEC and Müller cells. After treatment of the cells with $160 \mathrm{nM}$ PEDF for $24 \mathrm{~h}$, VEGF levels were dramatically decreased in both the RCEC and Müller cells (Fig. 1A). In RCEC exposed to hypoxia, PEDF has also shown a potent inhibitory effect on VEGF expression in a PEDF concentrationdependent manner (Fig. 1B).

\section{Positive feedback regulation of PEDF in retinal Müller cells}

Recombinant PEDF was added to and incubated with retinal Müller cells for $24 \mathrm{~h}$ in the presence of $200 \mu \mathrm{M}$ cobalt chloride $\left(\mathrm{CoCl}_{2}\right)$ or hypoxia $\left(<5 \% \mathrm{O}_{2}\right.$ concentration). The cells were harvested by trypsinization and thoroughly washed by PBS. The intracellular PEDF levels were determined by western blot analysis. The results showed that, after the $\mathrm{CoCl}_{2}$ (Fig. 2A) or hypoxia (Fig. 2B) treatment, the PEDF expression was drastically decreased. Treatment with exogenous PEDF significantly upregulated the expression of endogenous PEDF in a concentration-dependent manner (Fig. 2A, B). Western blot analysis showed that the PEDF treatment downregulated VEGF expression in the same samples (Fig. 2B).

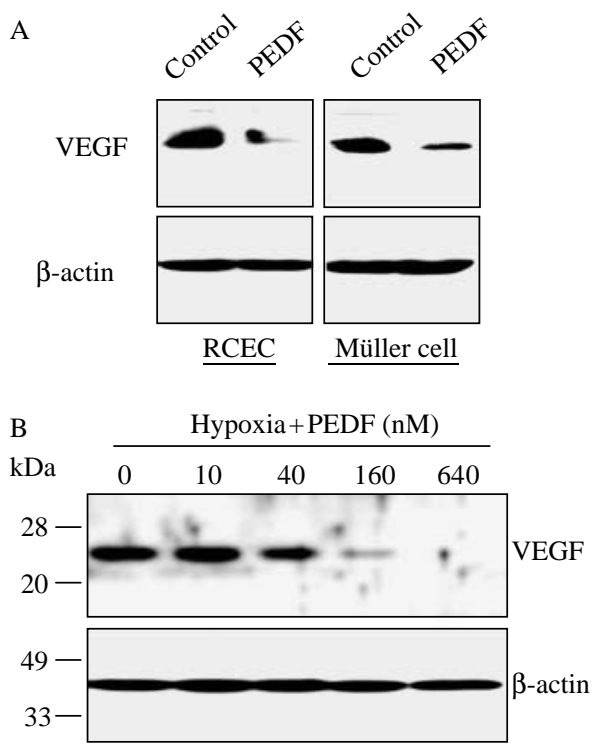

Figure 1 Inhibition of VEGF expression by PEDF in RCEC and Müller cells under normal and hypoxic condition. (A) RCEC and Müller cells were exposed to $160 \mathrm{nM}$ PEDF for $24 \mathrm{~h}$. The intracellular VEGF levels were determined by Western blot analysis. The same membrane was stripped and reblotted with an anti- $\beta$-actin antibody. (B) RCEC were exposed to $1 \%$ oxygen with different concentrations of PEDF for $24 \mathrm{~h}$. The VEGF levels in the cell lysate were determined by Western blot analysis. The same membrane was stripped and reblotted with the anti- $\beta$ actin antibody.
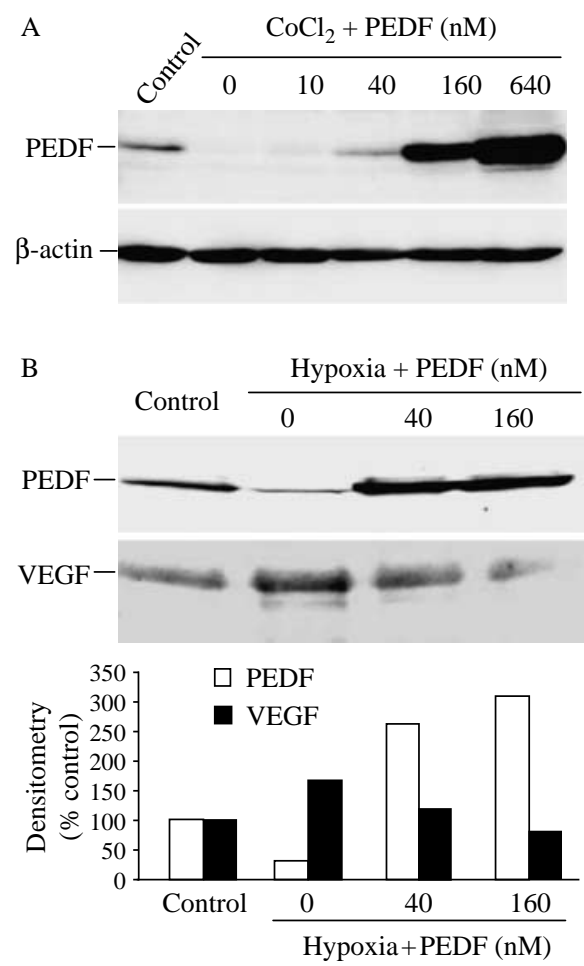

Figure 2 Autoregulation of PEDF and downregulation of VEGF by $P E D F$ in retinal Müller cells. (A) Müller cells were exposed to $200 \mu \mathrm{M} \mathrm{CoCl}_{2}$ with different concentrations of PEDF for $24 \mathrm{~h}$. The intracellular PEDF levels were determined by Western blot analysis. The same membrane was stripped and reblotted with the anti- $\beta$-actin antibody. (B) Cultured Müller cells were exposed to $1 \%$ oxygen with different concentrations of PEDF for $24 \mathrm{~h}$. The intracellular PEDF level was determined by Western blot analysis. The same membrane was stripped and reblotted with the antiVEGF antibody. The relative changes of PEDF and VEGF levels were semi-quantified by densitometry as shown in lower panel.

\section{PEDF upregulated endogenous PEDF and down- regulated VEGF expression in the retina of rats with OIR}

The effect of PEDF on the expression of endogenous PEDF and VEGF was determined in the retina of rats with OIR. The PEDF used for intravitreal injection was expressed and purified from Escherichia coli, which has a molecular weight of $45 \mathrm{kDa}$, lower than native PEDF $(50 \mathrm{kDa})$ due to the lack of glycosylation. At P14, PEDF was injected into the vitreous of one eye at $1 \mu \mathrm{g}$ /eye and PBS was injected into the contralateral eye as control. At $48 \mathrm{~h}$ after the injection, PEDF and VEGF levels in the retina were determined by western blot analysis. The results showed that endogenous PEDF levels in the retina were significantly higher in the PEDF-injected eyes than in the control eyes (Fig. 3A). Endogenous PEDF had a different molecular weight compared with the recombinant PEDF for the injection, indicating that the elevated retinal PEDF levels are indeed from endogenous expression. The VEGF levels, in contrast, 
A

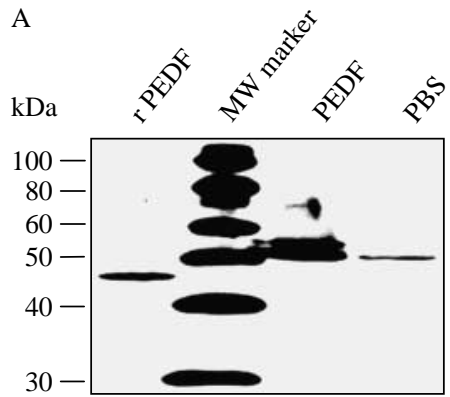

B

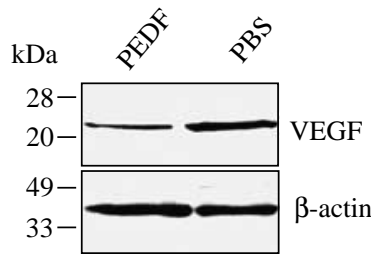

Figure 3 Autoregulation of PEDF and downregulation of VEGF by PEDF in the retina of rats with OIR. Newborn BN rats were exposed to $75 \%$ oxygen from age $\mathrm{P} 7$ to $\mathrm{P} 12$ and returned to room air to induce retinopathy. At P14, PEDF ( $1 \mu \mathrm{g} / \mathrm{eye})$ was injected intravitreally into one eye and PBS into the contralateral eye as control. At $48 \mathrm{~h}$ after the injection, the eyeballs were enucleated and the retinas were dissected. The PEDF levels $(A)$ and VEGF levels $(B)$ in the retina were determined by Western blot analysis. rPEDF, recombinant PEDF expressed and purified from E. coli.

were drastically decreased in the PEDF-injected eyes compared with the contralateral control eyes (Fig. 3B).

Silencing of PEDF increased VEGF secretion in cultured retinal Müller cells. At $24 \mathrm{~h}$ after PEDF siRNA transfection, RT-PCR showed that the PEDF mRNA was largely eliminated (Fig. 4A). The PEDF protein levels in the culture medium were also decreased as shown by ELISA (Fig. 4B). In the same cells, the mRNA and protein levels of VEGF were determined by quantitative real-time RT-PCR and ELISA respectively. The results showed that, after silencing of PEDF, the expression of VEGF was significantly increased at both the mRNA (Fig. 4C) and protein levels (Fig. 4D), suggesting that PEDF is an endogenous, negative regulator of VEGF expression in retinal Müller cells.

PEDF decreased VEGF promoter activity under hypoxic conditions but not under normoxic conditions. To further explore the mechanism responsible for the downregulation of VEGF expression by PEDF in the retina, we determined the effect of PEDF on the VEGF promoter activity in D407 cells. After exposing the transfected cells to $\mathrm{CoCl}_{2}$ for $6 \mathrm{~h}$, the VEGF promoter activity was significantly increased, indicating a hypoxiainduced activation in VEGF promoter (Fig. 5A, $P<0 \cdot 0001)$. PEDF at $160 \mathrm{nM}$ completely blocked the increase in VEGF promoter activity induced by $\mathrm{CoCl}_{2}$, suggesting that the downregulation of VEGF by PEDF in hypoxia is at least partially through the inhibition of VEGF gene transcription (Fig. 5A, $P<0 \cdot 0001$ ). Moreover, under normoxic conditions, PEDF had no effect on the VEGF promoter activity (Fig. 5A, $P>0 \cdot 15$ ), suggesting that PEDF plays an important role in maintaining the normal level of VEGF in the retina.

PEDF inhibits HIF- $1 \alpha$ activation and protein kinase phosphorylation. HIF- $1 \alpha$ is the major transcriptional factor mediating the regulation of VEGF expression (Hata et al. 1995). In cultured RCEC exposed to hypoxia with or without PEDF treatment for $24 \mathrm{~h}$, the nuclear and cytoplasmic proteins were isolated from the treated cells. Western blot analysis showed that, under normal culture conditions, HIF- $1 \alpha$ is almost undetectable in the nucleus (Fig. 5B). Exposure to hypoxia for $24 \mathrm{~h}$ resulted in a significant increase in the nuclear HIF-1 $\alpha$ level, indicating activation of HIF-1 induced by hypoxia (Fig. 5B). PEDF at 40 and $160 \mathrm{nM}$ dramatically inhibited the hypoxiainduced HIF-1 $\alpha$ nuclear translocation (Fig. 5B).

As the MAP kinase pathway is known to play a role in the VEGF regulation, we have determined the effect of PEDF on the activation of MAP kinase. Serum-deprived RCEC were stimulated with bFGF, and phosphorylation of $\mathrm{p} 42 / \mathrm{p} 44$ was determined by western blot analysis. A 5-min challenge with basic fibroblast growth factor (bFGF) resulted in a two-fold increase in p42/p44 phosphorylation when compared with those in the unchallenged cells (data not shown). PEDF decreased the bFGF-induced $\mathrm{p} 42 / \mathrm{p} 44$ phosphorylation by fourfold in RCEC (Fig. 5C). Similarly, we have also determined the effect of PEDF on MAP kinase activation in the retina. PEDF was injected into the vitreous of OIR rats at $\mathrm{P} 14$, and the retinas were dissected and pooled for MAP kinase phosphorylation assay at $48 \mathrm{~h}$ after the injection of PEDF. Consistent with the results in cultured RCEC, PEDF decreased p42/p44 phosphorylation two-fold in the retina of the rats with OIR (Fig. 5D).

PEDF competes with VEGF for binding to RCEC. Incubation of ${ }^{125}$ I-VEGF $(4 \mathrm{nM})$ with RCEC for $1 \mathrm{~h}$ resulted in specific binding of VEGF to VEGF receptor (VEGFR) on RCEC. To determine the competition between PEDF and VEGF for VEGFR binding, the same amount of ${ }^{125}$ I-VEGF was added to RCEC together with increasing concentrations $(40,400$, and $4000 \mathrm{nM})$ of unlabeled PEDF or plasminogen kringle 5 (K5), another potent angiogenic inhibitor. Unlabeled VEGF ( $400 \mathrm{nM}$ ) was used as a positive control. In the presence of excess amounts of unlabeled PEDF, VEGF bound to RCEC was decreased in a PEDF concentration-dependent manner (Fig. 6A). In contrast, $\mathrm{K} 5$ in the same concentration range did not inhibit VEGF binding with VEGFR, suggesting different mechanisms of action between PEDF and K5 (Fig. 6A), although they both reduce vascular permeability and inhibit retinal NV.

PEDF competed with VEGF for binding to VEGFR2 in vitro. To further explore whether the effect of PEDF on the blockade of VEGF binding to endothelial cells 
A
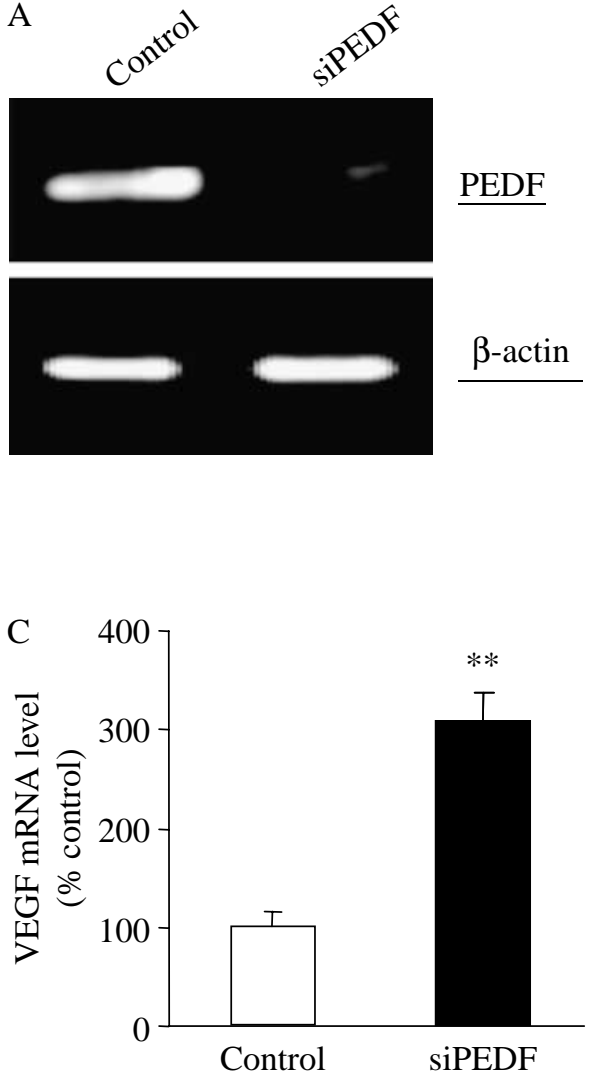

B
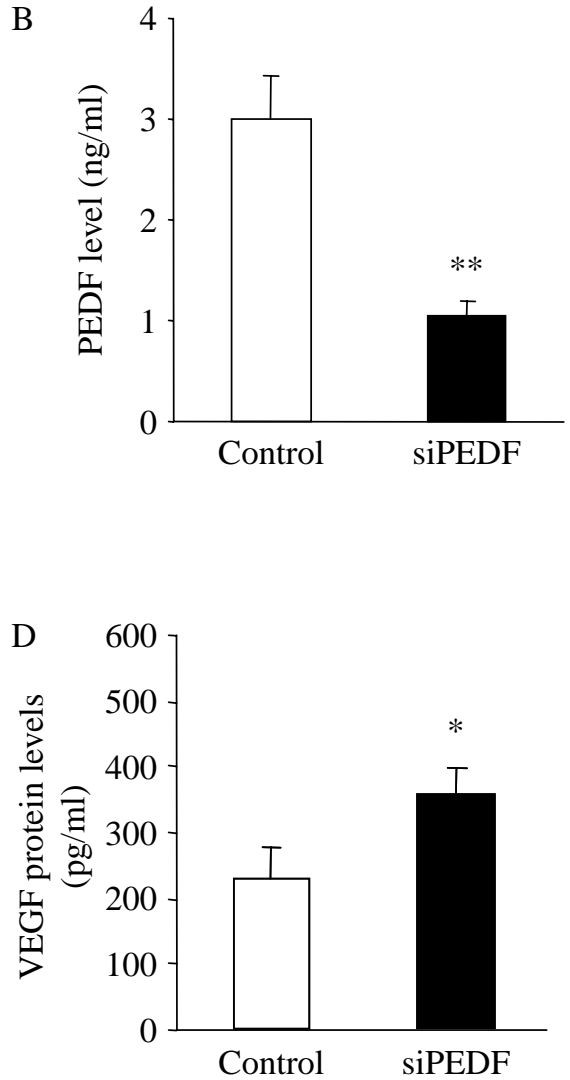

Figure 4 Increase in VEGF secretion after silencing of PEDF in retinal Müller cells. Müller cells were transfected with a PEDF siRNA or control, and cultured for $24 \mathrm{~h}$. (A). The mRNA level of PEDF in the cells was analyzed by RT-PCR and normalized by $\beta$-actin levels. (B). The PEDF secreted into the culture medium was measured by ELISA (mean \pm s.D., $n=3$ ). (C). The mRNA level of VEGF in the same cells was determined by real time RT-PCR and normalized by 18S RNA. The results were expressed as percentage of control (mean \pm S.D., $n=3$ ). (D). The protein levels of VEGF in the culture medium were measured by ELISA (mean \pm s.D., $n=3$ ). The values statistically different from the control are indicated by ${ }^{\star} P<0.05,{ }^{*} P<0 \cdot 01$.

is through the competition with VEGF for binding to the VEGF receptor, we performed an in vitro receptorbinding assay. VEGFR2, which is recognized as the major form of VEGF receptor responsible for the activity of VEGF in inducing vascular permeability and angiogenesis, was immobilized on a 96-well plate. The binding of VEGF to VEGFR2 was measured by an ELISA-based method (R\&D Systems). The results showed that the binding of VEGF to VEGFR2 was significantly inhibited by excess PEDF in a PEDF concentration-dependent manner (Fig. 6B). Moreover, the binding was not affected by the same concentrations of albumin (negative control) and heat-inactivated PEDF protein, suggesting that the competition of PEDF with VEGF on VEGFR2 binding is a specific effect (Fig. 6B).

VEGF decreased PEDF expression and secretion in RCEC but not in Müller cells. The effect of VEGF on the expression of PEDF was investigated in cultured RCEC and Müller cells. Western blot analysis showed that after 24-h incubation of RCEC with $40 \mathrm{ng} / \mathrm{ml}$ VEGF, the PEDF expression was drastically decreased (Fig. 7A). In retinal Müller cells, however, the same concentration of VEGF had no effect on the expression of PEDF (Fig. 7A). The same membrane was stripped and reblotted with an anti-VEGF antibody, which showed that VEGF induced a significant increase of endogenous VEGF expression in RCEC, but not in Müller cells (Fig. 7A and B), consistent with the cell type-specificity in its effect on PEDF expression. The effect of VEGF on the regulation of PEDF was further confirmed by measuring the PEDF secretion into the culture medium of RCEC. The ELISA result showed that VEGF decreased PEDF secreted from RCEC in a VEGF concentration-dependent manner (Fig. 7B). These results suggest that the effect of VEGF on the regulation of PEDF production and its autoregulation is endothelial cell-specific. 
A

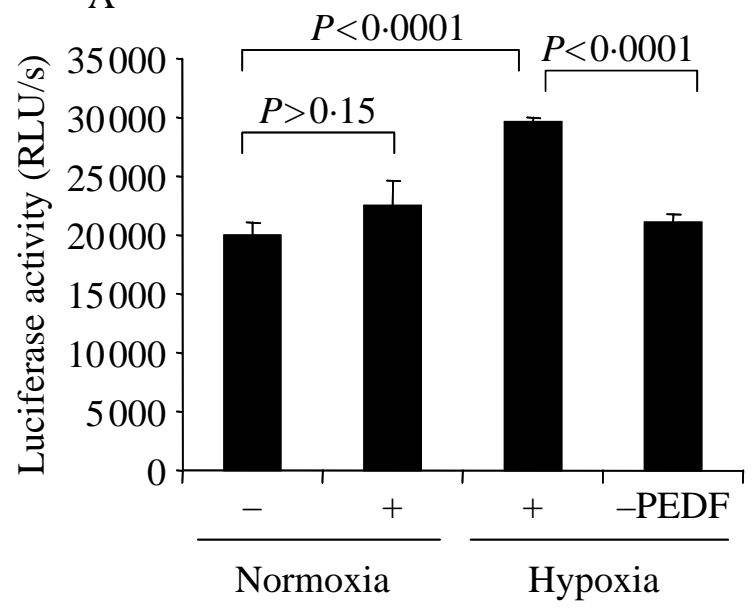

B

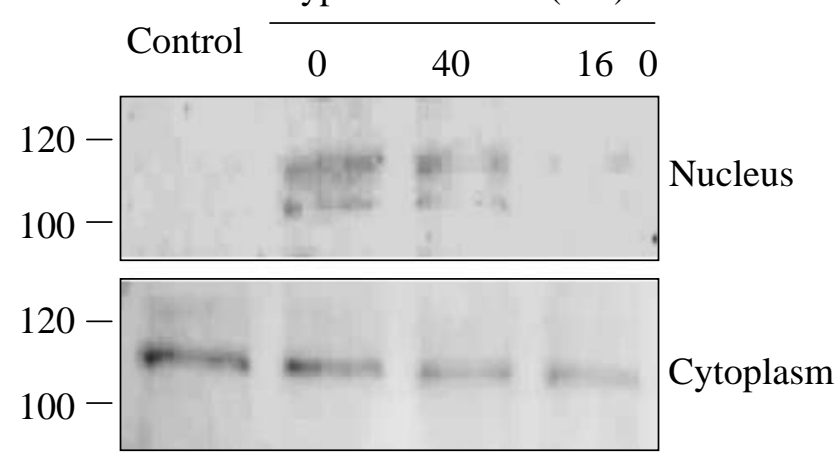

$\mathrm{C}$

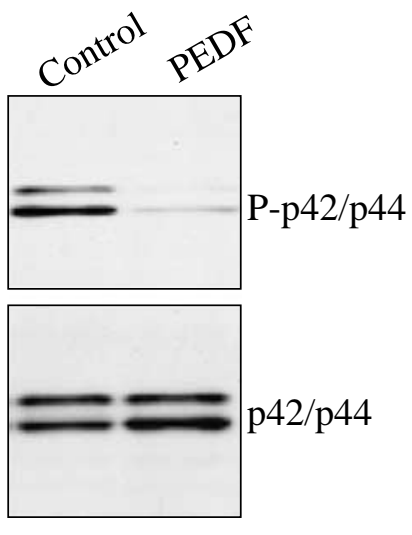

$\mathrm{D}$

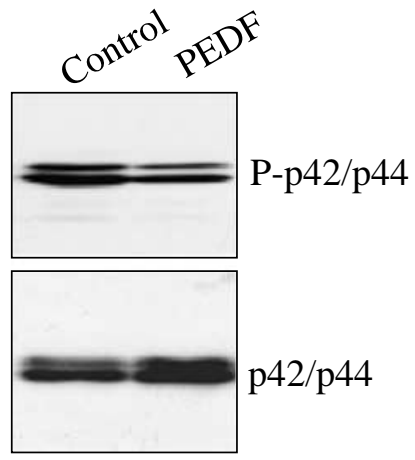

Figure 5 Inhibition of VEGF promoter activity, HIF-1 $\alpha$ nuclear translocation and MAP kinase phosphorylation by PEDF in cultured retinal cells and OIR retina. (A). D407 cells were transfected with a pGal3 vector containing the VEGF promoter and a luciferase reporter gene, in triplicate. Eighteen hours following transfection, the cells were exposed to $200 \mu \mathrm{M} \mathrm{CoCl}{ }_{2}$ in the presence or absence of $160 \mathrm{nM}$ PEDF for $6 \mathrm{~h}$. The luciferase activity was measured and normalized as described in Materials and methods. (B). Primary RCECs were exposed to $1 \%$ oxygen in the presence or absence of various concentrations of PEDF for $24 \mathrm{~h}$. The nuclear protein and cytoplasmic proteins were isolated from the cells. The HIF-1 $\alpha$ levels in the nucleus and in the cytoplasm were determined by Western blot analysis using the same amounts of proteins.

(C). Primary RCECs were stimulated with bFGF in the presence or absence of $160 \mathrm{nM}$ PEDF for $5 \mathrm{~min}$. The phosphorylated p42/p44 was semi-quantified by Western blot analysis using a phosphorylation-specific antibody and normalized by total p42/p44 levels. (D). At $48 \mathrm{~h}$ after the PEDF intravitreal injection in OIR rats, the phosphorylated and total p42/p44 levels in the retina were determined by Western blot analysis.

\section{Discussion}

In recent years, accumulating evidence suggests that the decrease of endogenous angiogenic inhibitors plays an important role in pathological vascular leakage and angiogenesis (Cao 2001, Gao et al. 2001, Boehm et al. 2003). PEDF, a serine proteinase inhibitor, has been found as a major anti-angiogenic factor in the eye (Dawson et al. 1999). High levels of PEDF in the transparent avascular ocular compartments, including the cornea and the vitreous, suggest its critical role in maintaining the local avascularity via its anti-angiogenic activity (Karakousis et al. 2001). In diabetic patients with active retinopathy, PEDF levels in the vitreous and aqueous humor are significantly decreased, which predicts the progression of retinopathy (Ogata et al. 2001b, 2002, Boehm et al. 2003). Our previous study demonstrated that in OIR rats, the decrease in PEDF and the increase in VEGF in the retina correlate with the elevation of vascular permeability and retinal NV, suggesting that a local balance between PEDF and VEGF is crucial for the regulation of retinal vascular function (Gao et al. 2001, Zhang et al. 2005b). The present study confirmed this finding by showing decreased PEDF expression and increased VEGF expression in hypoxia-exposed retinal Müller cells 

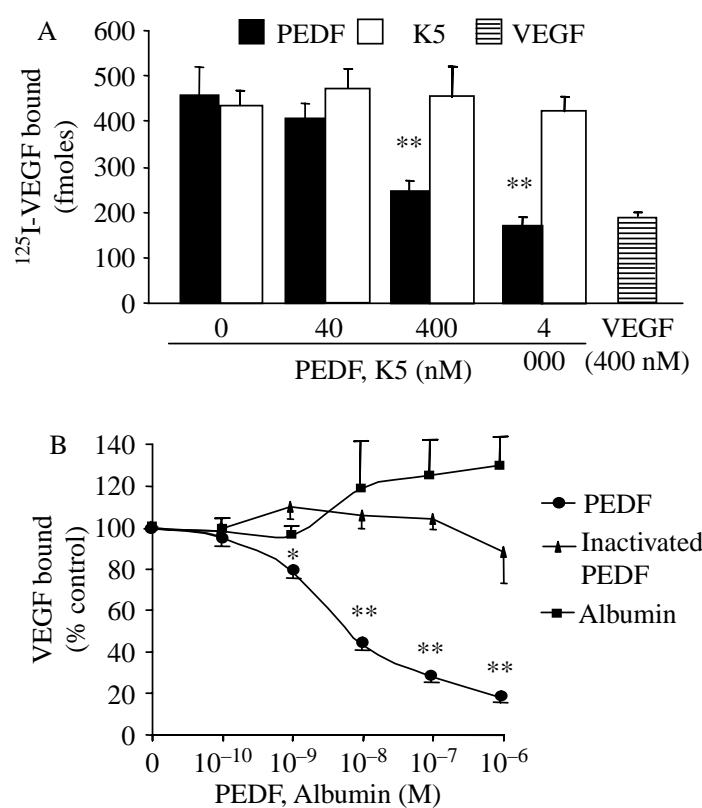

Figure 6 Blockade of the binding of VEGF to VEGFR2 by PEDF. (A) ${ }^{125}$ I-VEGF was incubated with primary RCEC in the absence and presence of excess amounts of PEDF or K5 as indicated. Unlabeled VEGF was used as a positive control. The binding of VEGF on RCEC was measured. Bars represent the bound VEGF (fmoles/well, means \pm s.D., $n=3$ ), and ${ }^{* \star}$ indicates the values statistically different from the control (VEGF alone) $(P<0.01)$. (B) Immobilized VEGFR2 was incubated with VEGF in the absence and presence of various concentrations of PEDF, heat-inactivated PEDF $\left(100^{\circ} \mathrm{C}\right.$ for $\left.5 \mathrm{~min}\right)$ or albumin. The binding of VEGF to VEGFR2 was detected as described in Materials and methods section. Values statistically different from the albumin control are indicated as ${ }^{\star} P<0 \cdot 05,{ }^{\star \star} P<0 \cdot 01$.

and RCEC. Although a number of studies have shown that oxygen concentration regulates the retinal levels of VEGF and PEDF, interactions between these two counterbalancing angiogenic factors are largely unclear (Gao et al. 2002a, Eichler et al. 2004, Werdich et al. 2004). The present study demonstrated for the first time that there is a reciprocal regulation between VEGF and PEDF in the retina. In cultured RCEC, PEDF downregulated hypoxia-induced VEGF expression at least partially via the inhibition of MAP kinase phosphorylation and HIF-1 nuclear translocation. In retinal Müller cells and in the retina of the OIR rats, PEDF upregulated the expression of endogenous PEDF. Moreover, silencing of PEDF alone significantly upregulated VEGF expression and secretion, suggesting that PEDF is an endogenous inhibitor of VEGF expression. In addition to the regulation of VEGF expression, PEDF effectively inhibited VEGF binding to retinal endothelial cells. In vitro studies demonstrated that PEDF competed with VEGF binding to VEGFR2. This competitive blockade of the VEGF-VEGFR2 binding may be responsible for the PEDF effects on
VEGF-induced permeability and angiogenesis. On the other hand, VEGF also significantly reduced PEDF expression and upregulated the expression of endogenous VEGF in endothelial cells, but not in Müller cells, suggesting a VEGF receptor-mediated regulation. These findings revealed a complex reciprocal regulation between PEDF and VEGF, which may be an important mechanism responsible for maintaining the balance between the angiogenic stimulator system and angiogenic inhibitor system in the retina.

VEGF is a potent VPF and a major angiogenic stimulator with endothelial cell-specific mitogenic activity and plays a crucial role in both normal and pathological angiogenesis (Dvorak et al. 1995, Pierce et al. 1995, Aiello 1996, Aiello et al. 1997). In diabetic patients with proliferative retinopathy, VEGF levels are significantly increased in the vitreous and the retina, and successful laser treatment decreased vitreous VEGF levels by $75 \%$ (Adamis et al. 1994, Aiello et al. 1994, Pe'er et al. 1995). In patients with DME, significantly elevated VEGF levels in the aqueous humor are correlated with the severity of macular edema (Funatsu et al. 2003). These findings suggest that over-production of VEGF in the retinal diabetics is a major cause of DME as well as retinal NV. In the retina, VEGF is produced by multiple cell types, including the RPE, pericytes, endothelial, glial, Müller, and ganglion cells (Pe'er et al. 1995, Dorey et al. 1996, Lu \& Adamis 2002). Among them, Müller cells and RPE are believed to be the major source of VEGF in the retina, and endothelial cells to be the primary target of VEGF (Pierce et al. 1995, Dorey et al. 1996). Cultured vascular endothelial cells also express high levels of VEGF in an autocrine manner (SimorrePinatel et al. 1994). In the present study, we have investigated the effect of PEDF on the regulation of VEGF expression in cultured retinal Müller cells and RCEC. Our results demonstrated that, under normoxic and hypoxic conditions, PEDF significantly downregulated VEGF expression in both RCEC and Müller cells as well as in the retina of the OIR rat model. Moreover, in cultured RPE cells, PEDF inhibited the hypoxiainduced increase in VEGF promoter activity, but had no effect on VEGF promoter activity under normoxic conditions. These results suggested that PEDF is a negative regulator of VEGF expression in the retina. In the normal eye, the high levels of PEDF in the vitreous and the retina inhibits the over-expression of VEGF, which, in turn, maintains the quiescence of retinal vascular endothelial cells and the integrity of BRB. Under hypoxia and other pathological conditions, in addition to the upregulation of VEGF induced directly by the primary insults, the decreased PEDF levels also contribute to the over-production of VEGF. The fact that silencing of PEDF gene expression alone is sufficient to increase VEGF expression further supports the role of PEDF as an endogenous inhibitor of VEGF 


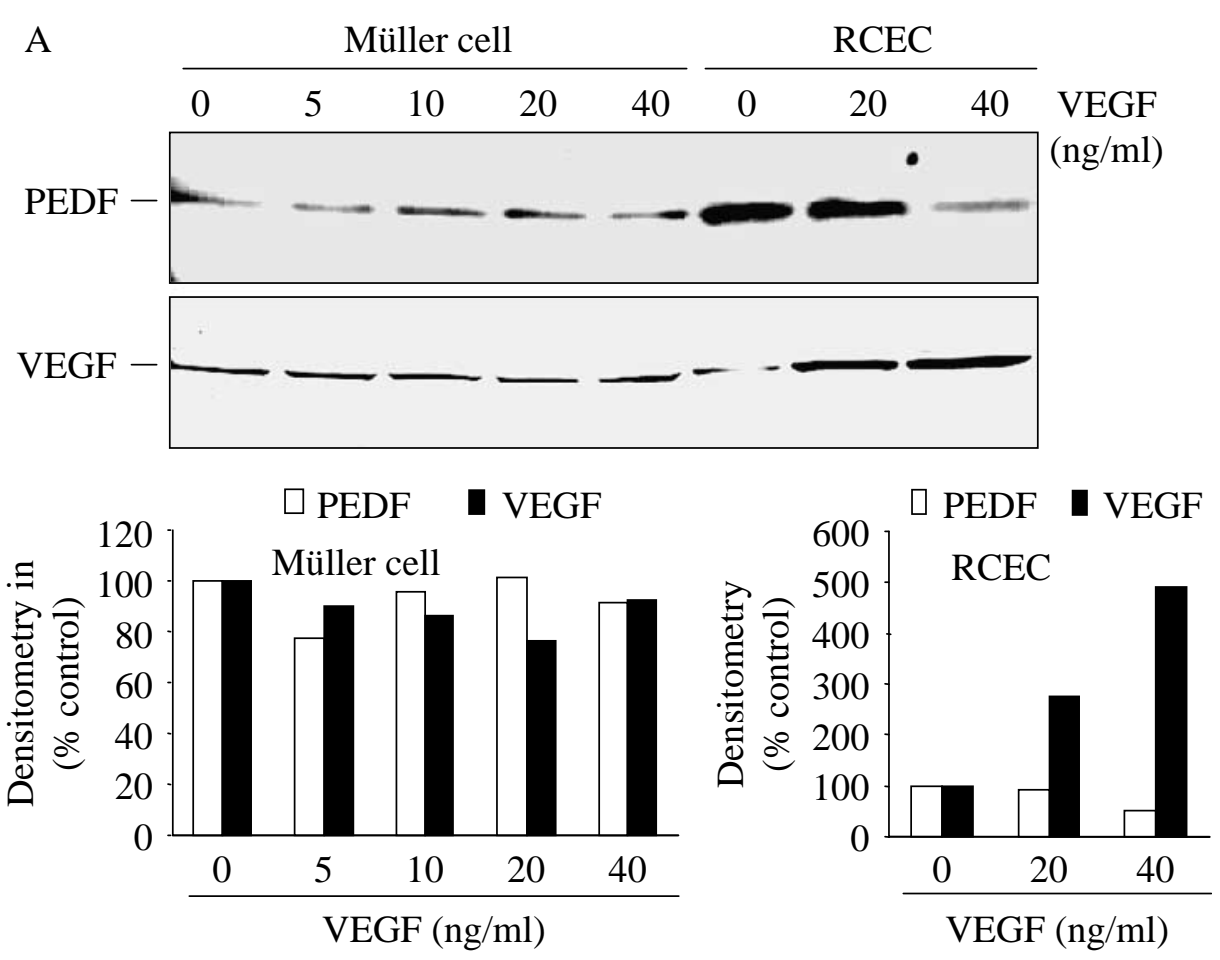

B

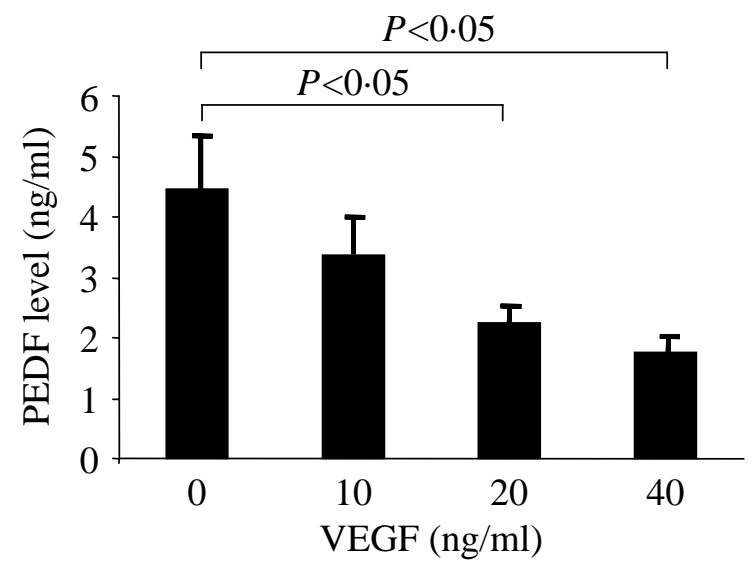

Figure 7 Inhibition of PEDF expression by VEGF in RCEC but not in Müller cells. RCEC and Müller cells were exposed to different concentrations of VEGF for $24 \mathrm{~h}$. (A). The intracellular PEDF levels were determined by Western blot analysis. The same membrane was stripped and reblotted with an antiVEGF antibody. The relative levels of PEDF and VEGF were semi-quantified by densitometry analysis. (B). The PEDF levels in the medium of cultured RCEC were measured by ELISA (mean \pm S.D., $n=3$ ).

expression and suggests that the reduced PEDF levels contribute to over-production and subsequent activation of vascular endothelial cells.

HIF-1 is a major transcriptional factor regulating VEGF expression under hypoxia (Forsythe et al. 1996, Ozaki et al. 1999). The present study demonstrated that the nuclear HIF- $1 \alpha$ level was elevated significantly in the retinal endothelial cells insulted by hypoxia, correlating with increased VEGF expression. Moreover, the total amount of HIF- $1 \alpha$ was drastically increased under hypoxic conditions (data not shown). Incubation with PEDF significantly reduced the nuclear HIF- $1 \alpha$ levels, indicating an inhibited HIF-1 nuclear translocation. These results suggest that inhibiting HIF-1 activation is responsible, at least partially, for the downregulated VEGF expression by PEDF. Furthermore, we determined if $\mathrm{p} 42 / \mathrm{p} 44$ MAP kinase activation, an important signaling pathway in VEGF expression, is involved in 
the regulation of VEGF by PEDF (Milanini et al. 1998). Our results demonstrated that PEDF significantly inhibited the activation of $\mathrm{p} 42 / \mathrm{p} 44$ in the retinal endothelial cells as well as in OIR retina, suggesting that the downregulation of VEGF by PEDF may be, at least in part, via inhibition of the MAP kinase activation. It is noteworthy that we have used a 24-h time scale in the experiments, as we found that the nuclear HIF-1 level was significantly higher at this time point. Although our results showed that PEDF inhibited MAPK activation and, thus, may directly block the downstream activation of HIF-1, we cannot exclude the possibility that this inhibitory effect of PEDF may be secondary, i.e. via blocking the positive feedback regulation of VEGF.

In addition to the regulation of VEGF expression, the present study, for the first time, demonstrated that PEDF effectively inhibited VEGF binding to VEGFR2 on RCEC and in isolated VEGFR2 in vitro. VEGFR2 is the major type of receptor responsible for the activity of VEGF on vascular permeability and angiogenesis (Aiello et al. 1995, Ozaki et al. 2000, Witmer et al. 2002). Several studies have shown that blockade of the activation of VEGFR2 induced by VEGF binding successfully abrogated retinal NV (Aiello et al. 1995, Ozaki et al. 2000). In the past decade, although PEDF has been well studied as a potent angiogenic inhibitor, the molecular mechanisms for its anti-angiogenic and anti-permeability activities are still uncertain. Our results demonstrated that PEDF significantly competed with the VEGF binding to VEGFR2, while the competitive capacity was lost in heat-inactivated PEDF. In endothelial cells, we have used an excess amount of PEDF to ensure the competitive effect of PEDF (Gotte et al. 2002). The structural basis responsible for the competition between PEDF and VEGF for binding to VEGFR2 remains to be elucidated.

PEDF is a multifaceted factor with neurotropic and anti-angiogenic functions (Tombran-Tink \& Barnstable 2003b, Tombran-Tink et al. 1991). Although $\mathrm{PEDF}$ as a major endogenous angiogenic inhibitor has been extensively studied, the regulation of PEDF is not yet well understood (Tombran-Tink \& Barnstable 2003b). Our study demonstrated that PEDF is secreted from RCEC and Müller cells. Under hypoxic conditions, PEDF secretion was significantly decreased along with the increase in VEGF expression. This result is consistent with the recent study reported by Eichler et al. (2004), which shows that hypoxia downregulates PEDF expression at the RNA level by $1 \cdot 7$-fold and at the protein level by two-fold in retinal Müller cells. Although hypoxia did induce a significant increase in VEGF expression and secretion, the addition of exogenous VEGF did not cause any change in the expression of PEDF in Müller cells, suggesting that hypoxia-induced downregulation of PEDF expression in Müller cells cannot be ascribed to the elevated VEGF levels. On the other hand, VEGF significantly downregulates PEDF expression in retinal endothelial cells, but not in Müller cells, suggesting that the regulation of PEDF by VEGF is dependent on VEGF receptor, which is expressed in endothelial cells but not in Müller cells.

Another important finding of the current study is that PEDF positively autoregulated endogenous PEDF expression in cultured Müller cells and in the retina of diabetic rats. PEDF is a major endogenous angiogenic inhibitor in the retina. Moreover, PEDF has recently been identified as a potent anti-inflammatory factor and anti-permeability factor (Liu et al. 2004, Zhang et al. 2005a). Furthermore, a decrease in ocular PEDF levels is believed to contribute to the pathogenesis of DR. Therefore, upregulation of endogenous PEDF levels in the retina will be beneficial in diabetic patients. The mechanisms by which PEDF is regulated by VEGF and by PEDF itself via positive feedback regulation remain to be determined in the future.

In summary, our study demonstrated that there exists a reciprocal regulation between PEDF and VEGF in the retina, which may represent an important mechanism for maintaining the balance between the two angiogenic regulatory systems. PEDF downregulates VEGF expression via inhibition of MAPK-mediated HIF-1 activation and competes with VEGF binding to VEGFR2. The decrease in PEDF levels is, at least partially, responsible for the increase in VEGF expression and the subsequent BRB breakdown and retinal NV in diabetic retinopathy. This study suggests that the angiogenic stimulator system and angiogenic inhibitor system have complex interactions, which may represent an important mechanism for maintaining the homeostasis of angiogenesis. The detailed mechanism responsible for these interactions remains to be elucidated.

\section{Acknowledgements}

This work was supported by NIH grants EY015650 and EY012231, JDRF research grant 1-2003-627 and a research award from ADA. The authors declare that there is no conflict of interest that would prejudice the impartiality of this scientific work.

\section{References}

Adamis AP, Miller JW, Bernal MT, D'Amico DJ, Folkman J, Yeo TK \& Yeo KT 1994 Increased vascular endothelial growth factor levels in the vitreous of eyes with proliferative diabetic retinopathy. American Journal of Ophthalmology 118 445-450.

Aiello LP 1996 Vascular endothelial growth factor and the eye. Past, present and future. Archives of Ophthalmology 114 1252-1254. 
Aiello LP \& Wong JS 2000 Role of vascular endothelial growth factor in diabetic vascular complications. Kidney International Supplement 77 S113-S119.

Aiello LP, Avery RL, Arrigg PG, Keyt BA, Jampel HD, Shah ST, Pasquale LR, Thieme H, Iwamoto MA \& Park JE 1994 Vascular endothelial growth factor in ocular fluid of patients with diabetic retinopathy and other retinal disorders. New England Journal of Medicine 331 1480-1487.

Aiello LP, Pierce EA, Foley ED, Takagi H, Chen H, Riddle L, Ferrara N, King GL \& Smith LE 1995 Suppression of retinal neovascularization in vivo by inhibition of vascular endothelial growth factor (VEGF) using soluble VEGF-receptor chimeric proteins. PNAS 92 10457-10461.

Aiello LP, Bursell SE, Clermont A, Duh E, Ishii H, Takagi C, Mori F, Ciulla TA, Ways K, Jirousek M et al. 1997 Vascular endothelial growth factor-induced retinal permeability is mediated by protein kinase $\mathrm{C}$ in vivo and suppressed by an orally effective beta-isoformselective inhibitor. Diabetes 46 1473-1480.

Antonetti DA, Barber AJ, Khin S, Lieth E, Tarbell JM \& Gardner TW 1998 Vascular permeability in experimental diabetes is associated with reduced endothelial occludin content: vascular endothelial growth factor decreases occludin in retinal endothelial cells. Penn State Retina Research Group. Diabetes 47 1953-1959.

Boehm BO, Lang G, Volpert O, Jehle PM, Kurkhaus A, Rosinger S, Lang GK \& Bouck N 2003 Low content of the natural ocular antiangiogenic agent pigment epithelium-derived factor (PEDF) in aqueous humor predicts progression of diabetic retinopathy. Diabetologia 46 394-400.

Bresnick GH 1986 Diabetic macular edema. A review. Ophthalmology 93 989-997.

Bussolino F, Mantovani A \& Persico G 1997 Molecular mechanisms of blood vessel formation. Trends in Biochemical Sciences 22 251-256.

Cao Y 2001 Endogenous angiogenesis inhibitors and their therapeutic implications. International Journal of Biochemistry and Cell Biology 33 357-369.

Dawson DW, Volpert OV, Gillis P, Crawford SE, Xu H, Benedict W \& Bouck NP 1999 Pigment epithelium-derived factor: a potent inhibitor of angiogenesis. Science 285 245-258.

Doll JA, Stellmach VM, Bouck NP, Bergh AR, Lee C, Abramson LP, Cornwell ML, Pins MR, Borensztajn J \& Crawford SE 2003 Pigment epithelium-derived factor regulates the vasculature and mass of the prostate and pancreas. Nature Medicine 9 774-780.

Dorey CK, Aouididi S, Reynaud X, Dvorak HF \& Brown LF 1996 Correlation of vascular permeability factor/vascular endothelial growth factor with extraretinal neovascularization in the rat. Archives of Ophthalmology 114 1210-1217.

Dvorak HF 2000 VPF/VEGF and the angiogenic response. Seminors in Perinatology 24 75-78.

Dvorak HF, Brown LF, Detmar M \& Dvorak AM 1995 Vascular permeability factor/vascular endothelial growth factor, microvascular hyperpermeability, and angiogenesis. American Journal of Pathology 146 1029-1039.

Eichler W, Yafai Y, Keller T, Wiedemann P \& Reichenbach A 2004 PEDF derived from glial Muller cells: a possible regulator of retinal angiogenesis. Experimental Cell Research 10 68-78.

Forsythe JA, Jiang BH, Iyer NV, Agani F, Leung SW, Koos RD \& Semenza GL 1996 Activation of vascular endothelial growth factor gene transcription by hypoxia-inducible factor 1. Molecular Cell Biology 16 4604-4613.

Frank RN 1991 On the pathogenesis of diabetic retinopathy. A 1990 update. Ophthalmology 98 586-593.

Funatsu H, Yamashita H, Ikeda T, Mimura T, Eguchi S \& Hori S 2003 Vitreous levels of interleukin-6 and vascular endothelial growth factor are related to diabetic macular edema. Ophthalmology 110 1690-1696.

Gao G \& Ma J 2002 Tipping the balance for angiogenic disorders. Drug Discovery Today 7 171-172.

Gao G, Li Y, Zhang D, Gee S, Crosson C \& Ma J 2001 Unbalanced expression of VEGF and PEDF in ischemia-induced retinal neovascularization. FEBS Letters 489 270-276.
Gao G, Li Y, Fant J, Crosson CE, Becerra SP \& Ma J-X $2002 a$ Difference in ischemic regulation of vascular endothelial growth factor and pigment epithelium-derived factor in brown Norway and SpragueDawley rats contributing to different susceptibilities to retinal neovascularization. Diabetes 51 1218-1225.

Gao G, Li Y, Gee S, Dudley A, Fant J, Crosson C \& Ma J-X $2002 b$ Downregulation of VEGF and up-regulation of PEDF: a possible mechanism for the anti-angiogenic activity of plasminogen kringle 5. Journal of Biological Chemistry 277 9492-9497.

Gao G, Shao C, Zhang SX, Dudley A, Fant J \& Ma J-x 2003 KallikreinBinding Protein: A Novel Angiogenic Inhibitor of the Serpin Family. Diabetologia 46 689-698.

Gotte M, Joussen AM, Klein C, Andre P, Wagner DD, Hinkes MT, Kirchhof B, Adamis AP \& Bernfield M 2002 Role of syndecan-1 in leukocyte-endothelial interactions in the ocular vasculature. Investigative Ophthalmology and Visual Science 43 1135-1141.

Hata Y, Nakagawa K, Ishibashi T, Inomata H, Ueno H \& Sueishi K 1995 Hypoxia-induced expression of vascular endothelial growth factor by retinal glial cells promotes in vitro angiogenesis. Virchows Archiv: An International Journal of Pathology 426 479-486.

Hatcher HC, Robinson EY \& Ma J-X 1998 Hypoxia induced proliferation of human retinal capillary endothelial cells but inhibited proliferation of human pericytes. Investigative Ophthalmology and Visual Science 39 S373.

Karakousis PC, John SK, Behling KC, Surace EM, Smith JE, Hendrickson A, Tang WX, Bennett J \& Milam AH 2001 Localization of pigment epithelium derived factor (PEDF) in developing and adult human ocular tissues. Molecular Vision 7 154-163.

Kim SY, Mocanu C, McLeod DS, Bhutto IA, Merges C, Eid M, Tong P \& Lutty GA 2003 Expression of pigment epithelium-derived factor (PEDF) and vascular endothelial growth factor (VEGF) in sickle cell retina and choroid. Experimental Eye Research 77 433-445.

Liu H, Ren JG, Cooper WL, Hawkins CE, Cowan MR \& Tong PY 2004 Identification of the antivasopermeability effect of pigment epithelium-derived factor and its active site. PNAS 101 6605-6610.

Lu M \& Adamis AP 2002 Vascular endothelial growth factor gene regulation and action in diabetic retinopathy. Ophthalmology Clinics of North America 15 69-79.

Milanini J, Vinals F, Pouyssegur J \& Pages G 1998 p42/p44 MAP kinase module plays a key role in the transcriptional regulation of the vascular endothelial growth factor gene in fibroblasts. Journal of Biological Chemistry 273 18165-18172.

Miller JW, Adamis AP \& Aiello LP 1997 Vascular endothelial growth factor in ocular neovascularization and proliferative diabetic retinopathy. Diabetes Metabolism Reviews 13 37-50.

Ogata N, Tombran-Tink J, Jo N, Mrazek D \& Matsumura M $2001 a$ Upregulation of pigment epithelium-derived factor after laser photocoagulation. American Journal of Ophthalmology 132 427-429.

Ogata N, Tombran-Tink J, Nishikawa M, Nishimura T, Mitsuma Y, Sakamoto T \& Matsumura M $2001 b$ Pigment epithelium-derived factor in the vitreous is low in diabetic retinopathy and high in rhegmatogenous retinal detachment. American Journal of Ophthalmology 132 378-382.

Ogata N, Nishikawa M, Nishimura T, Mitsuma Y \& Matsumura M 2002 Unbalanced vitreous levels of pigment epithelium-derived factor and vascular endothelial growth factor in diabetic retinopathy. American Journal of Ophthalmology 134 348-353.

Ohno-Matsui K, Morita I, Tombran-Tink J, Mrazek D, Onodera M, Uetama T, Hayano M, Murota SI \& Mochizuki M 2001 Novel mechanism for age-related macular degeneration: an equilibrium shift between the angiogenesis factors VEGF and PEDF. Journal of Cellular Physiology 189 323-333.

Ozaki H, Yu AY, Della N, Ozaki K, Luna JD, Yamada H, Hackett SF, Okamoto N, Zack DJ, Semenza GL et al. 1999 Hypoxia inducible factor-1alpha is increased in ischemic retina: temporal and spatial correlation with VEGF expression. Investigative Ophthalmology and Visual Science 40 182-189. 
Ozaki H, Seo MS, Ozaki K, Yamada H, Yamada E, Okamoto N, Hofmann F, Wood JM \& Campochiaro PA 2000 Blockade of vascular endothelial cell growth factor receptor signaling is sufficient to completely prevent retinal neovascularization. American Journal of Pathology 156 697-707.

Pe'er J, Shweiki D, Itin A, Hemo I, Gnessin H \& Keshet E 1995 Hypoxia-induced expression of vascular endothelial growth factor by retinal cells is a common factor in neovascularizing ocular diseases. Laboratory Investigation 72 638-645.

Pelzek C \& Lim JI 2002 Diabetic macular edema: review and update. Ophthalmology Clinics of North America 15 1-11.

Pierce EA, Avery RL, Foley ED, Aiello LP \& Smith LE 1995 Vascular endothelial growth factor/vascular permeability factor expression in a mouse model of retinal neovascularization. PNAS 92 905-909.

Qaum T, Xu Q, Joussen AM, Clemens MW, Qin W, Miyamoto K, Hassessian H, Wiegand SJ, Rudge J, Yancopoulos GD et al. 2001 VEGF-initiated blood-retinal barrier breakdown in early diabetes. Investigative Ophthalmology and Visual Science 42 2408-2413.

Shao C, Sima J, Zhang SX, Jin J, Reinach P, Wang Z \& Ma JX 2004 Suppression of corneal neovascularization by PEDF release from human amniotic membranes. Investigative Ophthalmology and Visual Science 45 1758-1762.

Simorre-Pinatel V, Guerrin M, Chollet P, Penary M, Clamens S, Malecaze F \& Plouet J 1994 Vasculotropin-VEGF stimulates retinal capillary endothelial cells through an autocrine pathway. Investigative Ophthalmology and Visual Science 35 3393-3400.

Tombran-Tink J, Chader GG \& Johnson LV 1991 PEDF: a pigment epithelium-derived factor with potent neuronal differentiative activity. Experimental Eye Research 53 411-414.
Tombran-Tink J \& Barnstable CJ $2003 a$ Therapeutic prospects for PEDF: more than a promising angiogenesis inhibitor. Trends in Molecular Medicine 9 244-250.

Tombran-Tink J \& Barnstable CJ $2003 b$ PEDF: a multifaceted neurotrophic factor. Nature Reviews Neuroscience 4 628-636.

Werdich XQ, McCollum GW, Rajaratnam VS \& Penn JS 2004 Variable oxygen and retinal VEGF levels: correlation with incidence and severity of pathology in a rat model of oxygen-induced retinopathy. Experimental Eye Research 79 623-630.

Witmer AN, Blaauwgeers HG, Weich HA, Alitalo K, Vrensen GF \& Schlingemann RO 2002 Altered expression patterns of VEGF receptors in human diabetic retina and in experimental VEGFinduced retinopathy in monkey. Investigative Ophthalmology and Visual Science 43 849-857.

Zhang SX, Sima J, Shao C, Fant J, Chen Y, Rohrer B, Gao G \& Ma J-x 2004 Plasminogen kringle 5 reduces vascular leakage in the retina of rat model of the oxygen-induced retinopathy and diabetes. Diabitologia 47 124-131.

Zhang SX, Wang JJ, Gao G, Shao C, Mott R \& Ma J-x 2005a Pigment epithelium-derived factor (PEDF) is an endogenous anti-inflammatory factor. FASEB Journal 20 323-325.

Zhang SX, Ma J-X, Sima J, Chen Y, Hu MS, Ottlecz A \& Lambrou GN $2005 \mathrm{~b}$ Genetic difference in susceptibility to the blood-retina barrier breakdown in diabetes and oxygen-induced retinopathy. American Journal of Pathology 166 313-321.

Received in final form 22 March 2006 Accepted 30 March 2006 